\title{
Efficient production of glycyrrhetinic acid in metabolically engineered Saccharomyces cerevisiae via an integrated strategy
}

\author{
Caixia Wang ${ }^{1+}{ }^{(0)}$, Xinyao $\mathrm{Su}^{1,2+}$, Mengchu Sun ${ }^{1,2}$, Mengting Zhang ${ }^{1,3}$, Jiajia Wu ${ }^{4}$, Jianmin Xing ${ }^{5}$, Ying Wang ${ }^{6}$,
} Jianping Xue ${ }^{2}$, Xia Liü ${ }^{3}$, Wei Sun ${ }^{1 *}$ and Shilin Chen ${ }^{1 *}$

\begin{abstract}
Background: Glycyrrhetinic acid (GA) is the most important ingredient in licorice due to its outstanding anti-inflammatory activity and wide application in the medicine and cosmetics industries. Contemporary industrial production of GA by acid hydrolysis of glycyrrhizin which was extracted from Glycyrrhiza plants, is not environment-friendly and devastates farmland since the Glycyrrhiza rhizomes grow up to $10 \mathrm{~m}$ underground.
\end{abstract}

Results: In this study, GA was produced through metabolically engineering Saccharomyces cerevisiae by introducing the entire heterogeneous biosynthetic pathway of GA. Codon optimized CYP88D6 and CYP72A154, combined with $\beta$-AS ( $\beta$-amyrin synthase encoding gene) and the NADPH-cytochrome P450 reductase gene of Arabidopsis thaliana were introduced into $S$. cerevisiae. The resulting strain (Y1) produced $2.5 \mathrm{mg} / \mathrm{L}$ of $\beta$-amyrin and $14 \mu \mathrm{g} / \mathrm{L}$ of $\mathrm{GA}$. The cytochrome 65 from G. uralensis (GUCYB5) was identified and the introduction of this novel GUCYB5 increased the efficiency of GA production by eightfold. The joint utilization of the GUCYB5 gene along with 10 known MVA pathway genes from S. cerevisiae were overexpressed in a stable chromosome integration to achieve higher GA production. Using the combined strategy, GA concentration improved by 40-fold during batch fermentation. The production was further improved to $8.78 \mathrm{mg} / \mathrm{L}$ in fed-batch fermentation, which was increased by a factor of nearly 630 .

Conclusions: This study first investigated the influence of carbon flux in the upstream module and the introduction of a newly identified GUCYB5 on GA production. The newly identified GUCYB5 was highly effective in improving GA production. An integrated strategy including enzyme discovery, pathway optimization, and fusion protein construction was provided in improving GA production, achieving a 630 fold increase in GA production. The metabolically engineered yeast cell factories provide an alternative approach to glycyrrhetinic acid production, replacing the traditional method of plant extraction.

Keywords: Glycyrrhetinic acid, Triterpene, Saccharomyces cerevisiae, Metabolic engineering

\footnotetext{
*Correspondence: tcmsunwei@outlook.com; slchen@icmm.ac.cn

${ }^{\dagger}$ Caixia Wang and Xinyao Su contributed equally to this work

${ }^{1}$ Key Laboratory of Beijing for Identification and Safety Evaluation

of Chinese Medicine, Institute of Chinese Materia Medica, China Academy

of Chinese Medical Sciences, No. 16 Nanxiaojie, Dongzhimennei Ave,

Beijing 100700, People's Republic of China

Full list of author information is available at the end of the article
} 


\section{Background}

Chinese licorice, Glycyrrhiza, is a leguminous plant species whose roots and rhizomes have been widely used in herbal medicine and as a natural sweetener. More than 200 active ingredients were identified in Glycyrrhiza uralensis of which glycyrrhizin (GL) and glycyrrhetinic acid (GA) are the major bioactive triterpenoid saponins [1]. Chemically, GL is made up of one GA as aglycone and two D-glucuronic acid [2, 3], with a wide range of pharmacological activities. These include anti-inflammatory [4], immunomodulatory, and antiviral, specifically against different DNA and RNA viruses such as human immunodeficiency virus (HIV) and severe acute respiratory syndrome (SARS)-associated coronavirus $[2,3,5]$. Clinically, GL is metabolized into GA after oral administration, which then becomes the bioactive molecule in vivo $[6,7]$. Indeed, GA has been widely used in both the cosmetic and pharmaceutical industry for its whitening and anti-inflammatory effect [8].

The high market price of GA, which is nearly twice that of GL, necessitates methods for direct GA production. However, chemical synthesis of GA is not feasible because of its structural complexity. The low GA content in licorice, as precursor of GL, also makes the direct extraction of GA from plants not cost effective. Currently, GA is produced by acid hydrolysis of GL, which accounts for $2-8 \%$ of the dry weight of licorice [9]. This acid consuming hydrolysis procedure is inefficient and expensive with regard to the consequent environment pollution. On the other hand, the raw materials of GL are mainly extracted from Glycyrrhiza plants whose cultivation is time intensive, requiring 3-4 years to reach higher GL content. Deep growth of Glycyrrhiza roots and rhizomes, up to $10 \mathrm{~m}$, underground also results in farmland devastation and eventual desertification after plant harvest, making GA production from Glycyrrhiza cultivation unsustainable.

Rapid development of functional genomics has accelerated the identification of key genes involved in the biosynthesis of natural products [10-12]. This has enabled de novo production of valuable bioactive molecules in
Escherichia coli or S. cerevisiae using synthetic biology and metabolic engineering [10, 12]. The growing list of natural products that have been synthesized in recent years includes artemisinin [13, 14], protopanaxadiol [15], ginsenosides [16, 17], ginkgolides [18], vindoline [19], tanshinones [20, 21], strictosidine [22], and gastrodin [23]. For example, the complete biosynthetic pathway of artemisinic acid was engineered and optimized in S. cerevisiae [13], resulting in $25 \mathrm{~g} / \mathrm{L}$ of artemisinic acid production.

As a typical oleanane-type triterpenoid, GA is synthesized from isopentenyl diphosphate (IPP) and dimethylally diphosphate (DMAPP), two intermediates of the mevalonate pathway (MVA) that presents within the cytoplasm and mitochondria of all plant cells. The cyclization of 2, 3-oxidosqualene to $\beta$-amyrin catalyzed by $\beta$-amyrin synthase is the first reaction towards GA biosynthesis [24]. After this site-specific oxidation at positions $\mathrm{C}-11$ and $\mathrm{C}-30, \beta$-amyrin are catalyzed by two cytochrome P450 enzymes, CYP88D6 and CYP72A154, leading to the formation of GA $[9,25]$. Subsequently, GA is converted to GL through a recently identified UDPdependent glucuronosyltransferases, GuUGAT [26]. The complete gene set for GA biosynthesis makes GA production in yeast possible (Fig. 1a).

Considering the expanding GA demand and the limited Glycyrrhiza resource supply, our objective was to produce GA from glucose using engineered S. cerevisiae. As a precursor of GA, $\beta$-amyrin was first produced in yeast by Keasling's group [27], with a resulting concentration of $6 \mathrm{mg} / \mathrm{L}$. It was further improved to $138 \mathrm{mg} / \mathrm{L}$ by Li' s group [28]. We finally improved it into $158 \mathrm{mg} / \mathrm{L}$ now (data is under review). From $\beta$-amyrin to GA, it needs two cytochrome P450 enzymes, CYP88D6 and CYP72A154, making the synthesis of GA difficult in yeast. Recently, Li's group successfully achieved glycyrrhetinic acid synthesis in S. cerevisiae and boosted its concentration by introducing efficient cytochrome P450s (CYP450s: Uni25647 and CYP72A63) and pairing their reduction systems [29]. However, only influence of carbon flux in downstream module (the below

\footnotetext{
(See figure on next page.)

Fig. 1 a Biosynthetic pathway of glycyrrhetinic acid constructed in S. cerevisiae. IPP: isopentenyl pyrophosphate; DMAPP: dimethylallyl pyrophosphate; GPP: geranyl diphosphate; FPP: farnesyl diphosphate; GGPP: geranylgeranyl pyrophosphate; ERG10: acetyl-CoA C-acetyltransferase; ERG13: hydroxymethylglutaryl-CoA synthase; tHMG1: truncated 3-hydroxy-3-methylglutaryl-CoA reductase 1; ERG12: mevalonate kinase; ERG8: phosphomevalonate kinase; ERG19: diphosphomevalonate decarboxylase; IDI1: dimethylallyl diphosphate isomerase; ERG20: Farnesyl pyrophosphate synthetase; ERG9: squalene synthase; ERG1: squalene epoxidase; $\beta$-AS: $\beta$-amyrin synthase; AtCPR1: cytochrome P450 reductase from Arabidopsis thaliana; CYP88D6: $\beta$-amyrin 11-oxidase; CYP72A154: 11-oxo-beta-amyrin 30-oxidase; b Biosynthetic pathway of ergosterol in S. cerevisiae. BTS1: arnesyltranstransferase; ERG7: lanosterol synthase; ERG11: sterol 14-demethylase; ERG24delta(14)-sterol reductase; ERG25: methylsterol monooxygenase; ERG26: sterol-4-alpha-carboxylate 3-dehydrogenase; ERG27: 3-keto-steroid reductase; ERG28: ergosterol biosynthesis 28; ERG2: C-8 sterol isomerase; ERG3: C-5 sterol desaturase; ERG4: delta(24(24(1)))-sterol reductase; ERG5: C-22 sterol desaturase; ERG6: sterol 24-C-methyltransferase
} 

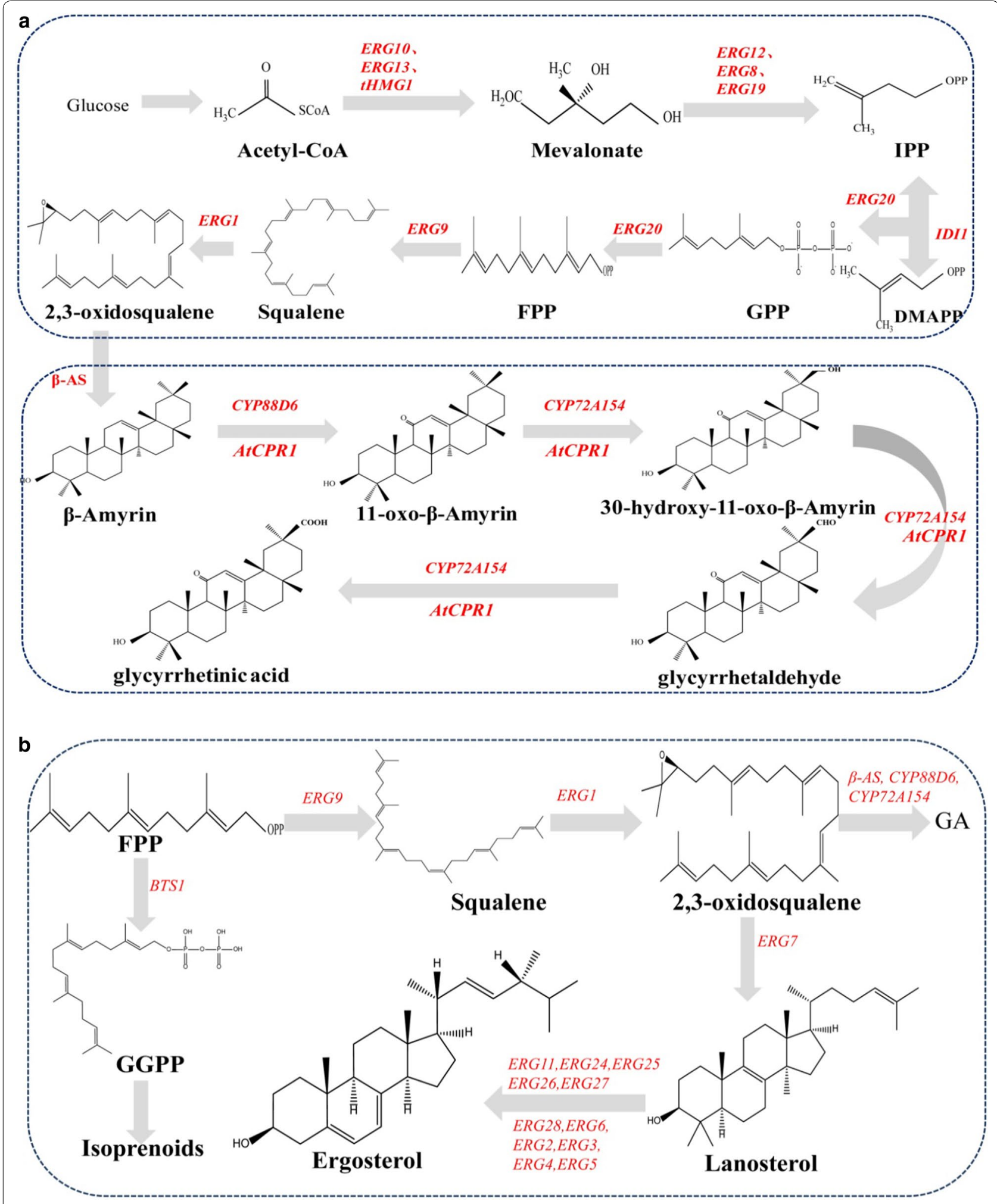
box in Fig. 1a) on GA production was investigated in that study. Besides CYP450s and CPR1, the carbon flux flowing into MVA pathway from acetyl-CoA, especially into the synthesis of 2,3-oxidosqualene, is crucial to the production of GA. The pipeline of carbon flux flowing into 2,3-oxidosqualene in MVA pathway determined how high that GA's production can reach. Thus, detailed influence of carbon flux in upstream module of GA should be investigated. Cytochrome b5 genes were believed as an another important factor that facilitates the expression of CYP450s, as it can provide an electron for P450 oxidation. For example, with the identification of CYB5 from Artemisia annua, the production of artemisinic acid was improved sharply in S. cerevisiae.

Based on the background, we first integrated heterologously GA formation genes into the chromosome of the $S$. cerevisiae to create the GA-producing strain. Then, we investigated native upstream module factors of MVP that likely regulate GA biosynthesis. We used transcriptome mining to identify cytochrome $b 5$, which was capable of facilitating GA production. Lastly, we constructed a first generation GA-producing S. cerevisiae (Fig. 1a). Our study provides an efficient solution for GA production in yeast, and sets a solid foundation for other triterpenoids biosynthesis using metabolically engineered yeast.

\section{Results}

\section{Construction of the glycyrrhetinic acid synthetic pathway in S. cerevisiae}

Overall, the flowchart of yeast strain construction in this study were shown in Additional file 1: Fig. S1.

We attempted to construct the metabolic pathway for GA production in $S$. cerevisiae. The four gene cassettes of $\beta$-AS, CYP88D6, CYP72A154, and AtCPR1 were first integrated into the chromosome of $S$. cerevisiae Cen.pk2$1 \mathrm{D}$ at the rDNA site. Six positive colonies (Y0-1,2,3,4,5,6) were randomly selected and verified by PCR amplification. Analysis of cell extracts by GC-MS confirmed the successful production of $\beta$-amyrin and GA in these constructed strains ranging $0.02-1.35 \mathrm{mg} / \mathrm{L}$ for $\beta$-amyrin and 6.1-12.6 $\mu \mathrm{g} / \mathrm{L}$ for GA (Additional file 1: Fig. S2A, B).

We then optimized the codons of CYP88D6 and $C Y P 72 A 154$, and integrated them together with $\beta-A S$ and AtCPR1 into S. cerevisiae Cen.pk2-1D. Also, six colonies $(\mathrm{Y} 1-1,2,3,5,8)$ were selected and verified by PCR amplification. Concentrations of $\beta$-amyrin and GA of these six strains were shown in Additional file 1: Fig. S2C, D. Based on the results, strain Y1-8 (designed as Y1) gave the best performance. The highest concentrations of $\beta$-amyrin (Fig. 2) and GA (Fig. 3) produced by strain Y1 improved to $17 \mathrm{mg} / \mathrm{L}$ and $19.5 \mu \mathrm{g} / \mathrm{L}$, respectively (Fig. 4a, b).

Fermentation of $\mathrm{Y} 1$ and the blank control S. cerevisiae Cen.pK2-1D was conducted in YPD media with $2 \%$ glucose as the carbon source. While the control clone cannot synthesize $\beta$-amyrin and GA, its lanosterol concentration is about 140 -fold that of $\mathrm{Y} 1$ at approximately the same level of ergosterol but only $20 \%$ of squalene levels (Fig. 4c-e). Considering the fact that in yeast sterol (lanosterol and ergosterol) and GA share the same precursor of squalene, these results suggest that the introduction of the GA synthetic pathway in S. cerevisiae Cen. pk2-1D consumed the squalene pool and decreased the carbon flux into sterol.

\section{Overexpressing genes in MVA pathway for improving glycyrrhetinic acid production}

In eukaryotic cells, farnesyl pyrophosphate (FPP), squalene and 2,3-oxidosqualene are the three consecutive precursors of GA. As the branching point, FPP that is produced from IPP and DMAPP by ERG20 can be converted to either GGPP by BTS1 or squalene by ERG9. A previous study demonstrated that the over-expression of BTS1 and fusion expression of ERG20-BTS1 channeled the FPP flux to miltiradiene production rather than to other FPP-consuming pathways [20]. This implies that the loss of function for BTS1 should favor the formation of squalene, thus improving GA production.

It was also reported that over-expression of a truncated HMG-CoA reductase gene (tHMG1) effectively improved triterpoind production $[15,16]$ while overexpression of the mevalonate pathway (MVP) genes in S. cerevisiae CEN.PK2-1D doubled artemisinic acid production [13]. Thus, we evaluated the over-expression effects of ten MVA genes on GA production.

\section{Overexpression of $E R G 20, E R G 9, E R G 1$ and $t H M G 1$ on the production of glycyrrhetinic acid}

To channel FPP flux to squalene production, EG2O and ERG9 were fused in ERG20-ERG9 and ERG9-ERG20 two configurations, driven by constitutive TDH3 promoters and terminated by CYC1 terminators. Different configurations may have different effect on enhancement of squalene pool which was crucial for GA production. Thus, ERG20-ERG9 fused gene was integrated into the delta sites of strain $Y 1$, together with expression cassettes of $E R G 1$ and $t H M G 1$, resulting Y2 series strains $(Y 2-1,2,3,4,5)$. Similar integration was adopted for ERG9-ERG20 gene cassette, giving Y3 series strains (Y3-1,2,3,4,5). GA concentration was further analyzed for these positive colonies. It turned out that the ERG9ERG20 clone Y3-1 (designed as Y3) had the highest GA concentration but the ERG20-ERG9 clone Y2-4 (designed as Y2) had higher average GA content (Additional file 1: Fig. S3), both of which were approximately $20 \%$ higher than Y1 (Fig. 4a). It's noteworthy that 
x10 6 Authentic: 1:Squalene; 2: Ergosterol; 3: Lanosterol; 4: $\beta$-Amyrin

a

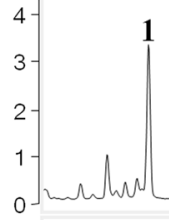
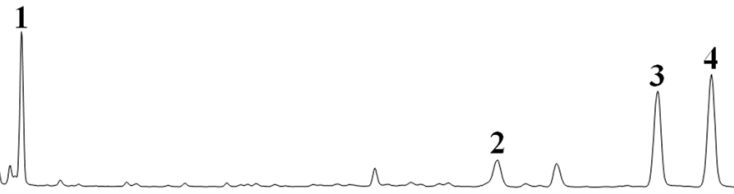

C10 ${ }^{7}$ Control sample : Cen.pk2-1D

b

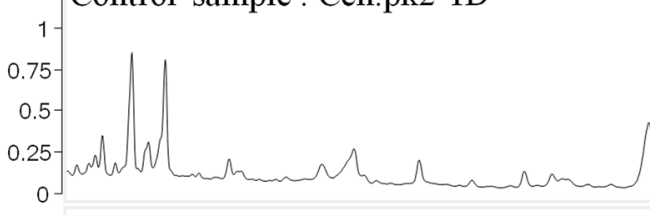

$\times 10^{7}$ Sample:Y1-8

C
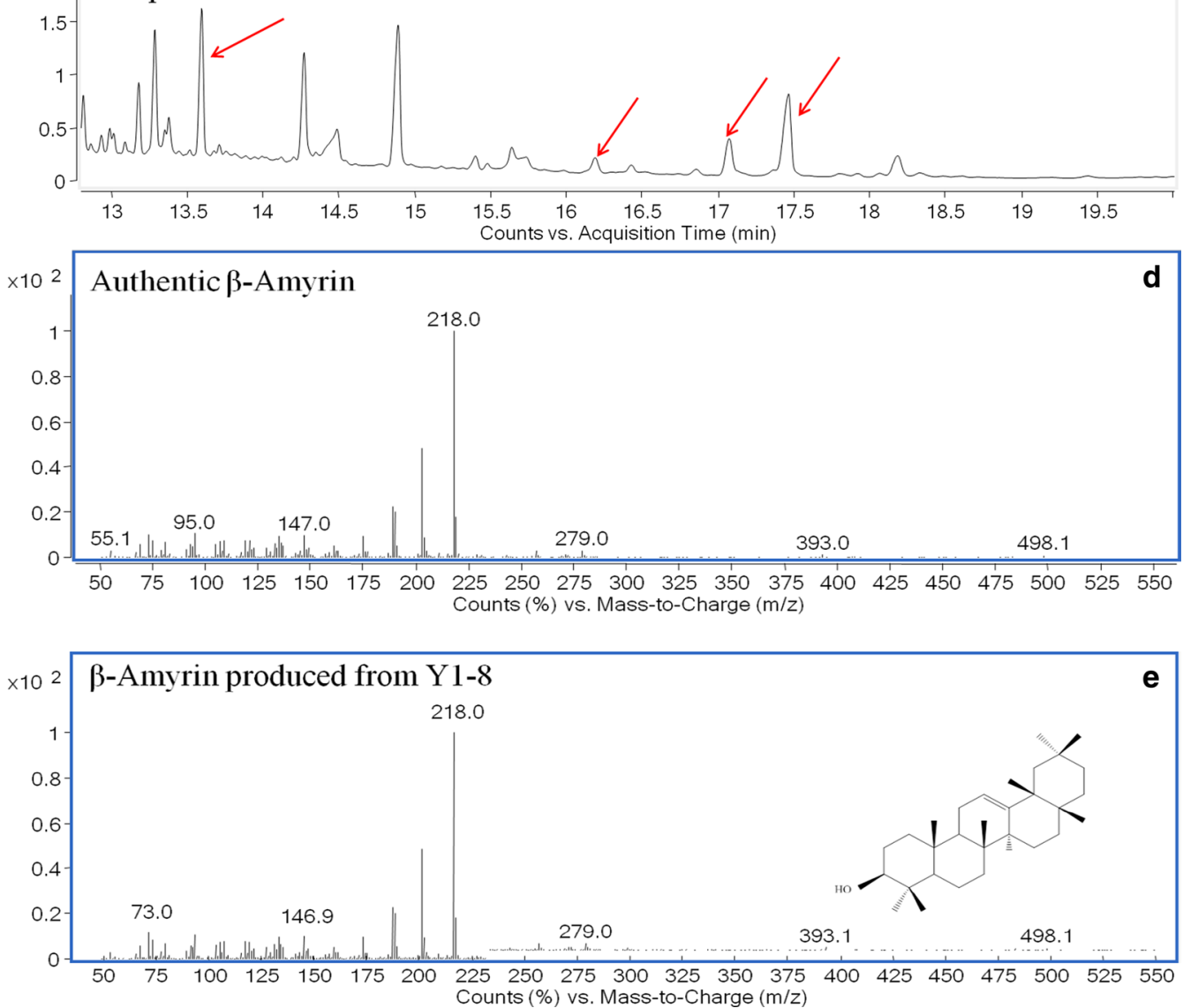

Fig. 2 Fermentation product identification for strain Y1-8; a GC-MS analysis of (1) squalene, (2) ergosterol, (3) lanosterol, (4) $\beta$-amyrin standards; b GC-MS analysis of cell extraction of control strain Cen.pk 2-1D; $\mathbf{c}$ GC-MS analysis of cell extraction of constructed strain Y1-8; d mass spectra of $\beta$-amyrin standard; e mass spectra of $\beta$-amyrin produced from strain $Y 1-8$ 

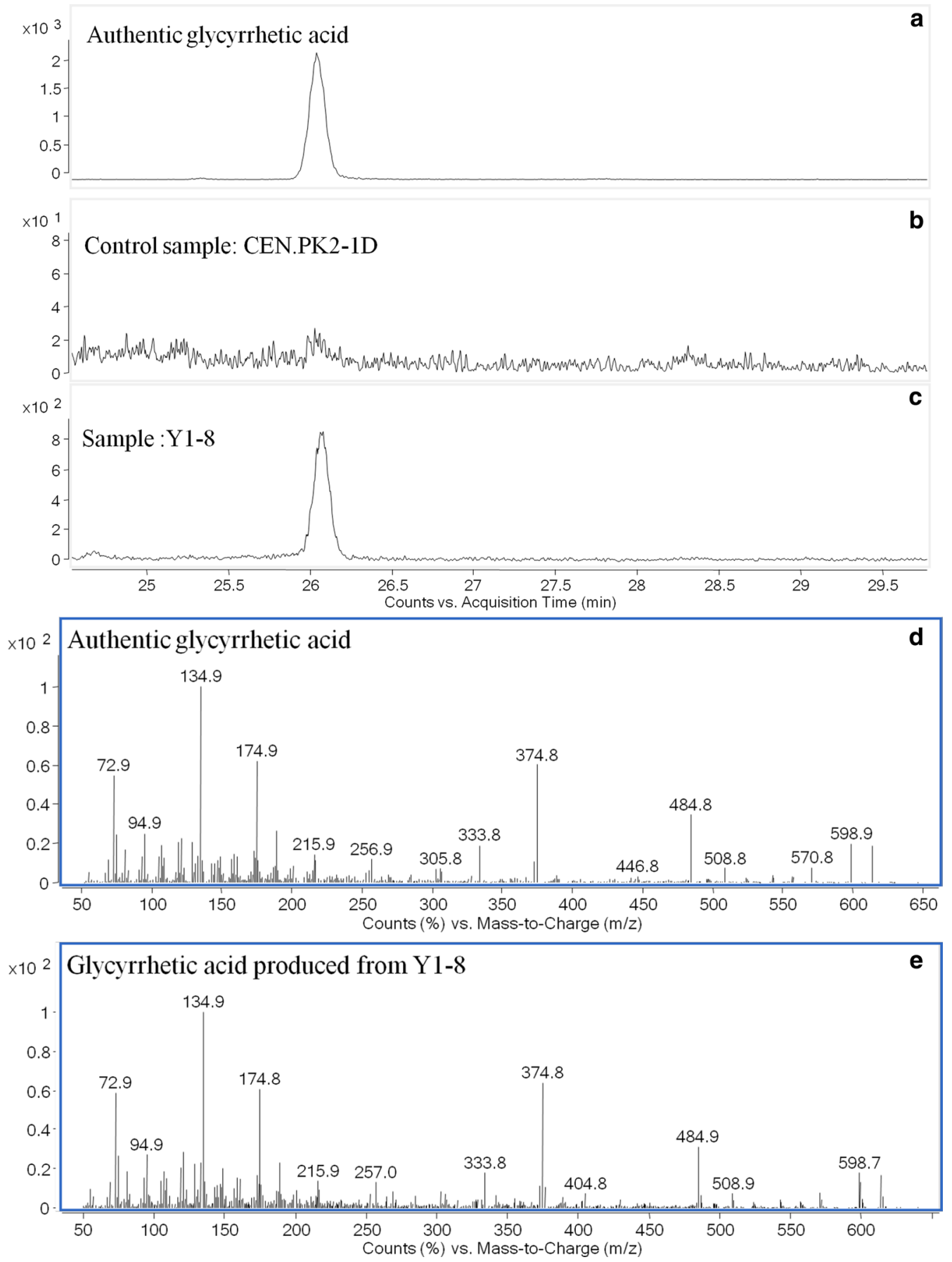

Fig. 3 De novo glycyrrhetic acid production from strain Y1-8; a GC-MS analysis of glycyrrhetic acid standards; $\mathbf{b}$ GC-MS analysis of cell extraction of control strain CEN.PK2-1D; c GC-MS analysis of cell extraction of constructed strain Y1-8; $\mathbf{d}$ mass spectra of glycyrrhetic acid standard; $\mathbf{e}$ mass spectra of glycyrrhetic acid produced from strain $\mathrm{Y} 1$ 


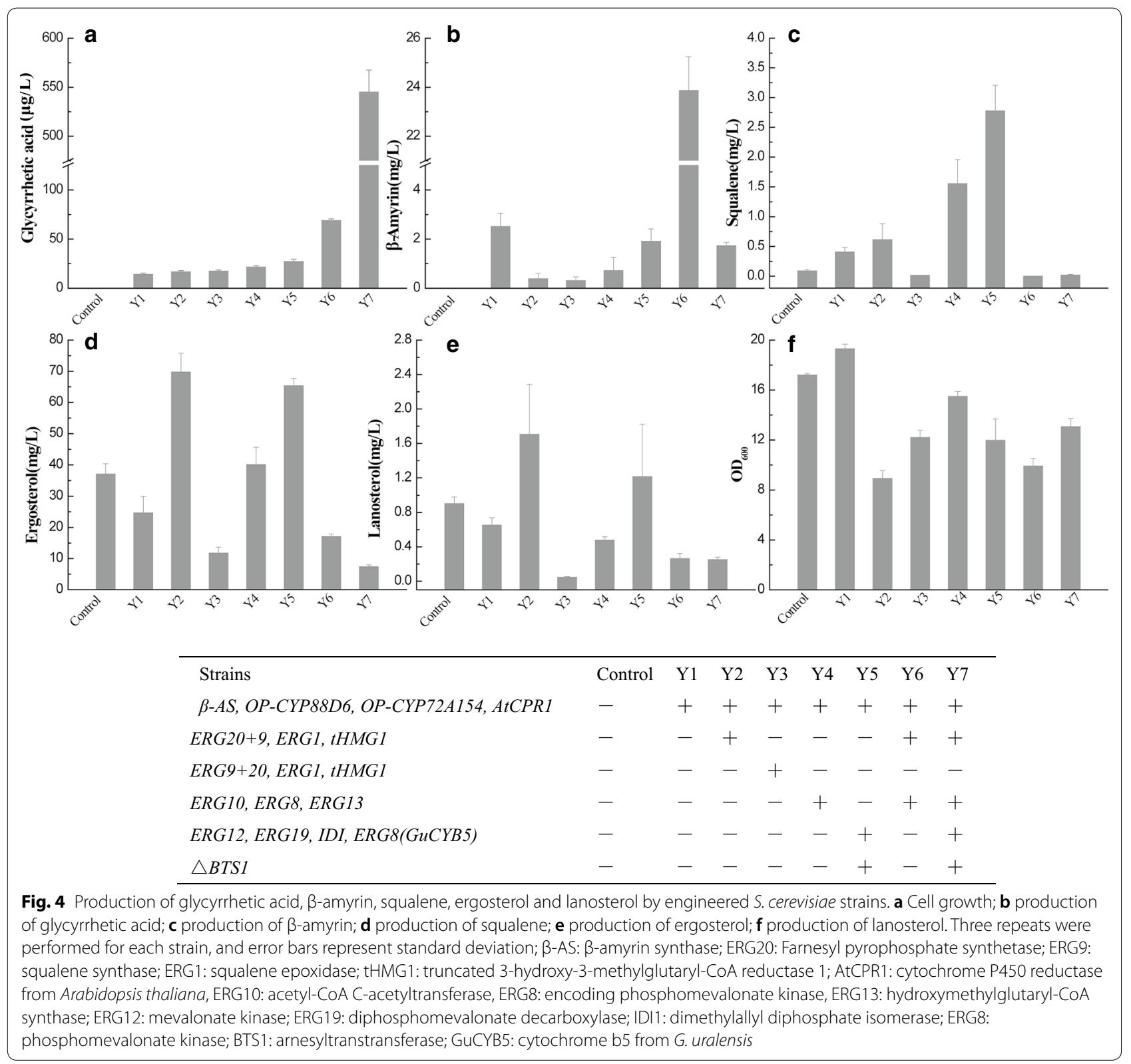

different configuration of fusion resulted in distinct concentrations of squalene, ergosterol, lanosterol, all of which were higher than Y1, suggesting that the carbon source was mostly directed to sterol biosynthesis rather than GA production upon the integration of $E R G 20+9$, ERG1 and $t H M G 1$.

\section{Overexpression of ERG10, ERG8, ERG13, ERG12, ERG19, IDI1 on the production of glycyrrhetinic acid}

We further evaluated the effects of the upstream genes of the MVP pathway on GA production. ERG10 (encoding acetyl-CoA C-acetyltransferase), ERG8 (encoding phosphomevalonate kinase), and ERG13 (encoding hydroxymethylglutaryl-CoA synthase) were integrated into the YDR007W site of strain Y1. As YDR007W site presents only one copy in the genome of $S$. cerevisiae, one positive colony was verified and designed as strain Y4 whose genotype can be seen in Table 2. Squalene, ergosterol and GA of strain Y4 increased $2.78,1.66$, and 1.5 fold, respectively, while lanosterol $(0.48 \mathrm{mg} / \mathrm{L})$ and $\beta$-amyrin $(0.71 \mathrm{mg} / \mathrm{L})$ concentrations were about $17 \%$ and $48 \%$ that of Y1. It seemed that unlike the ERG20+9, ERG1 and $t H M G 1$ over-expression, the ERG10, ERG8, and ERG13 cassette increased squalene and GA production without interfering sterol biosynthesis. 
Similarly, gene expression cassettes of ERG12 (encoding mevalonate kinase), ERG19 (encoding diphosphomevalonate decarboxylase), IDI1 (encoding isopentenyl diphosphate isomerase) and ERG8 (encoding phosphomevalonate kinase) were inserted into the YPL069C site flanking the truncated BTS1 (encoding farnesyltranstransferase) gene (Fig. 1b). Since BTS1 catalyzes the conversion of FPP to GGPP, this construct, designated as strain Y5, should channel more carbon from FPP to squalene production (Fig. 1). Indeed, squalene of Y5 improved dramatically from 0.41 to $2.8 \mathrm{mg} / \mathrm{L}$, and $\beta$-amyrin decreased from 2.5 to $1.9 \mathrm{mg} / \mathrm{L}$ (Fig. $4 \mathrm{~b}, \mathrm{c}$ ). The final concentration of GA of Y5, being twice that of Y1 (Fig. 4a), demonstrated the effectiveness of this design.

\section{Integrated strategy for improved glycyrrhetinic acid production}

Based on the results in "Overexpression of ERG20, ERG9, $E R G 1$ and $t H M G 1$ on the production of glycyrrhetinic acid" and "Overexpression of ERG10, ERG8, ERG13, ERG12, $E R G 19, I D I 1$ on the production of glycyrrhetinic acid", we selected strain Y4 with ERG10, ERG8, and ERG13 integration as the basis for further metabolic engineering. First, ERG20-ERG9, ERG1, and tHMG1 were integrated into the delta sites of Y4. By this way, clones Y6(1-8) were obtained. Variation within GA concentration of eight random colonies demonstrated the integration of different copy numbers of focal genes (Additional file 1: Fig. S4). Of these colonies, Y6-1 (designed as Y6) yielded five-fold GA than that of Y1, with undetectable squalene as well as lower ergosterol and lanosterol concentration, suggesting that this protocol may efficiently direct carbon flux to GA production.

A recent study has demonstrated that cytochrome b5 (CYB5) can provide electrons to its interacting partner cytochromes P450 (P450), enhancing P450's enzymatic activities. For example, over-expression of cytochrome b5 from A. annua resulted in a 3.5 fold increase of artemisinic acid and $40 \%$ increase of total sesquiterpene production [13]. In this study, we identified a CYB5 gene of G. uralensis (Additional file 1: Fig. S5) and assembled GuCYB5 using the TDH3 promoter and transformed it into the YPL069C site of the Y6 strain (designated as Y7). Not surprisingly, GA concentration of Y7 reached $545 \mu \mathrm{g} / \mathrm{L}$, which was about 8 times that of $\mathrm{Y} 6$ and 40 fold more than Y1 (Fig. 4a). Additionally, a decrease of $\beta$-amyrin by $25 \%$ and lower concentrations of squalene and lanosterol was observed (Fig. 4b-d). Since sterol and triterpenoid share the same carbon flux from glucose to squalene, increased GA concentration of Y7 demonstrated that carbon flux was successfully channeled to triterpenoid biosynthesis rather than sterol.

We monitored the growth of these constructed yeast strains (Fig. 4f) and found that strain Y2 and Y6, both with higher GA production, grew slower than the control strain while the remaining strains showed similar growth rates. This seemed to suggest that intracellular accumulation of GA and its precursors encumbered normal growth of yeast. The apparent normal growth of $Y 7$ under high GA concentration, on the other hand, may be attributed to the function of $G u C Y B 5$, as reported in previous artemisinic acid work [13].

\section{Scale-up fed-batch fermentation of GA production}

To further improve GA production, fed-batch fermentation was conducted in a $7.5 \mathrm{~L}$ fermentor. Since metabolites accumulate inside the cell, GA production is highly correlated with cell density in that higher cell density increases GA production. Thus, we monitored growth of strain $\mathrm{Y} 7$ on different mediums before fed-batch fermentation (Additional file 1: Fig. S6). Maximum strain growth $\left(\mathrm{OD}_{600}=17.36\right)$ was obtained with the fermentation medium as expected. Fed-batch fermentation of strain $\mathrm{Y7}$ in fermentation medium was conducted and results were shown in Fig. 5. Strain growth was much faster than that in the flask, as the $\mathrm{OD}_{600}$ was 10 times higher than that in the flask, with a maximum of 175 . Final GA concentration reached $8.78 \mathrm{mg} / \mathrm{L}$, which was nearly 630 -fold higher than that produced by the original strain. The concentration of $\beta$-amyrin was $12.8 \mathrm{mg} / \mathrm{L}$, which was a moderate level. However, the produced 11-oxo- $\beta$-amyrin, the direct GA precursor, significantly increased to $80 \mathrm{mg} / \mathrm{L}$ (data not shown). The total triterpenoid produced in the study reached to $101 \mathrm{mg} / \mathrm{L}$. Replicate fed-batch fermentation attained GA of $6.9-7.8 \mathrm{mg} / \mathrm{L}$. Our results illustrate an effective method for improving GA and 11-oxo- $\beta$-amyrin production.

\section{Discussion}

Recently, much attention has been given to the synthesis of natural products utilizing microorganisms via fermentation since the process is green and sustainable [30, 31]. We selected S. cerevisiae for the production of plant

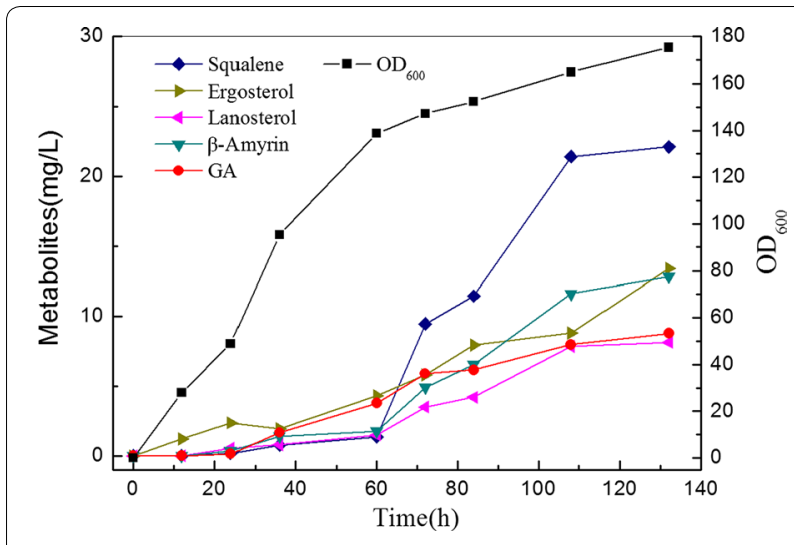

Fig. 5 Time profile of fed-batch GA production in a $7.5 \mathrm{~L}$ fermentor 
derived terpenoids, as it provides a similar physical and physiological intracellular environment for the functional expression of cytochrome P450 (CYP450s) and uridine diphosphate glycosyltransferases (UGTs) from plants and allows for endomembrane localization and post-translational modifications.

In this study, we produced glycyrrhetinic acid using the metabolic engineering of $S$. cerevisiae by introducing the entire heterogeneous biosynthetic pathway of GA. With the overexpression of ERG20, ERG9, ERG1 and tHMG1, we were able to improve GA production by approximately $21 \%$, although this was lower than reported in previous studies. The over expression of tHMG1 significantly increased protopanaxadiol production between 10 and 90 fold depending upon the gene copies integrated in the chromosome of S. cerevisiae [15]. The integration of ERG20, ERG9 and PgSQE1 (squalene epoxidase 1 encoding gene from Panax ginseng) increased protopanaxadiol by 3.3 times [17]. Although protopanaxadiol and GA share the same two precursors, 2 and 3-oxidosqualene, the integration of $t H M G 1$ did not improve GA production to the same extent as protopanaxadiol. Despite an limited increase in GA production, levels of precursors including squalene, ergosterol, and lanosterol of Y2 were much higher than Y1, specifically $50 \%, 188 \%$ and $161 \%$, respectively. This suggests that the improved metabolic flux in the MVA pathway of strain Y2 by the integration of ERG20-ERG9, ERG1 and $t H M G 1$ were likely directed to the sterol synthesis, and partially to GA synthesis. This finding is supported by previous studies, which also reported the transcription level of ERG2O and ERG9 increased 3.3- and 2.8-fold in overexpressed ERG20 and ERG9 strains for $\beta$-amyrin production in the $\beta$-amyrin producing strain [28]. Our results showed a $66 \%$ increase of $\beta$-amyrin in the Y2 strain compared to strain Y1. We believe that the increase in $\beta$-amyrin through the integration of ERG20-ERG9, tHMG1, and ERG1 was partially catalyzed to form both 11-oxo- $\beta$-amyrin and GA, leading to lower increases of $\beta$-amyrin. Constructing protein fusion can be highly effective in enhancing the metabolic flux channeled to the synthesis pathway of desired products by engineering their active sites to a closer proximity [20]. In this study, the fusion of ERG20 and ERG9 was constructed to direct the FPP flux to squalene production with two modules of ER20-ERG9 and ERG9-ERG20. Results indicated that the fusion of ER20-ERG9 enhanced the conversation of FPP to squalene although more carbon flux was directed into sterol.

The low yield of natural functional ingredients produced by microorganisms was mainly due to low catalytic activities of the introduced plant cytochrome P450 enzymes [32, 33]. The poor coupling between cytochrome P450 and CPR may have lead to the release of reactive oxygen species, decreasing cell growth and product yields [13, 34]. Previous findings have shown that $C Y B 5$ facilitates and in some cases was required for the reduction of $\mathrm{P} 450$ enzymes by providing an electron for P450 oxidation (Additional file 1: Fig. S7) [35-37]. The introduction of newly identified GuCYB5 in this study was highly efficient in improving GA production as indicated by our results. With AtCPR1 providing the electron for CYP450, oxidation was less efficient than CPR1 derived from G. uralensis (GuCPR1) in GA production [29]. The limited coupling between CYP88D6, $C Y P 72 A 154$ and AtCPR1 resulted in a relatively lower GA yield, and highlighted the effectiveness of GuCYB5, which significantly improved the production of GA. Therefore, future work should focus on balancing the expression between CYP88D6, CYP72A154, and CPR for heightened optimization due to the importance for cell growth and product synthesis. Formerly, GuCPR1 was identified as presenting the highest electron transfer efficiency among several related CPRs from different plant sources recently [29]. Additionally, future work should include self-sufficient P450 reduction enzyme construction to further increase GA production efficiency.

The production level of precursors was around milligram per liter, but GA concentration was only microgram per liter in flask fermentation. In the fed-batch fermentation process, we found that 11 -oxo- $\beta$-amyrin reached nearly $80 \mathrm{mg} / \mathrm{L}$. These results demonstrate that rate-limiting steps exist in the 11-oxo- $\beta$-amyrin to GA conversion. There are two CYP450 genes responsible for catalyzing $\beta$-amyrin to GA: CYP88D6 and CYP72A154, of which CYP72A154 is a transmembrane protein (Additional file 1: Fig. S8). From this, the ratelimiting step in GA production was likely the final step catalyzed by CYP72A154 and investigating the protein engineering of CYP72A154 is imperative. We deduce that $\mathrm{N}$-terminal amino acid residues used to truncate CYP72A154 will improve GA production, and further systematic investigation is needed. Like $C Y P 71 A V 1$ in $A$. annua, CYP72A154 mostly catalyzes 11 -oxo- $\beta$-amyrin to form GA, leading to the intermediate formation of 30-hydroxy-11-oxo- $\beta$-amyrin and glycyrrhetaldehyde (Fig. 1a). An identification of aldehyde dehydrogenase and alcohol dehydrogenase from G. uralensis is also needed to improve the catalytic capacity of CYP72A154. Further work addressing these aspects of GA production will enhance GA production like artemisinic acid and strictosidine producing strain construction [13, 22].

Like other triterpenoids produced by yeast, GA is reconstituted in yeast at relatively lower yields, as supported by Zhu et al. [29]. It is important to mention that for the original strains, Zhu et al. used a diploid strain while we used a haploid strain making the 
effectiveness of strategies employed to improve GA production comparable. Specifically, Zhu et al. focused on the optimization of the CYP450 gene expression and the reduction systems where this study investigated the influence of carbon flux in the upstream module and introduction of GuCYB5 on GA production. The genetic strategy provided by Zhu's study combined with the workflow presented in this study would likely further improve GA production.

Here, we report a S. cerevisiae strain capable of producing GA, where improvement of GA production was achieved using a combined strategy comprised of enzyme discovery, pathway optimization, and fusion protein construction. A newly identified gene from G. uralensis, together with 10 known MVA pathway genes from S. cerevisiae were overexpressed in a stable chromosome integration to achieve higher GA creation. Utilizing these methods we were able to show a 40-fold increase of GA attaining $0.5 \mathrm{mg} / \mathrm{L}$ in a flask batch fermentation condition. GA production was further improved to $8.78 \mathrm{mg} / \mathrm{L}$ by fed-batch fermentation, 630 times higher than the parent strain. These results provide insights into the influence of the MVA carbon flux on $\beta$-Amyrin based triterpeniods. More importantly, we show the effectiveness of GuCYB5 in improving GA production. In short, this study provides an effective integrated strategy for GA production using yeast, to achieve a 630 fold improvement in GA production. This strategy as well as the GAproducing strain provides solid foundation for future production of other triterpenoids in yeast.

\section{Conclusions}

The codon optimizated CYP88D6 and CYP72A154, together with the $\beta-A S$ and $A t C P R 1$ were integrated into $S$. cerevisiae, to construct a glycyrrhetinic acid producing yeast. A novel $G u C Y B 5$ gene, which enhances the reaction rate of $C Y P 88 D 6$ and $C Y P 72 A 154$, was identified from G. uralensis. Over expression of different genes in the MVA pathway showed positive influences on GA production, varying from 0.21 to 5 fold increases in GA production. However, GuCYB5 was found to be the most effective in improving GA production, specifically 8 times more effective. Through the integration of genes attributed to the MVA pathway with $G u C Y B 5$, GA production reached a max of $0.55 \mathrm{mg} / \mathrm{L}$ during flask fermentation, which was further improved to $8.78 \mathrm{mg} / \mathrm{L}$ in fed-batch fermentation, allowing for a 630 fold increase of the parent strain Y1. The constructed yeast strains may provide an alternative source for glycyrrhetinic acid or $\beta$-amyrin based triterpenoids and reduce the need to extract from licorice.

\section{Materials and methods}

\section{Prediction and cloning of CYB5 of $G$. uralensis}

By using the CYB5 (AtCYB5) protein sequence from Arabidopsis thaliana as a reference, we were able to identify CYB5 sequence from G. uralensis utilizing the website http://ngs-data-archive.psc.riken.jp/Gur/blast.pl (accessed on 2017-3-30). To clone GuCYB5, we used the primers found in Additional file 1: Table S1.

\section{Vector construction}

Total RNA was isolated from the fresh roots of $G$. uralensis using the RNA prep pure Plant Kit (TIANGEN, Beijing, China). We obtained cDNA of G. uralensis using the Prime-Script 1st Strand cDNA Synthesis Kit (Takara, Dalian, China). To amplify $\beta-A S, C Y P D D 6$, and CYP72A154 from the cDNA of the G. uralensis, we used the primers in Additional file 1: Table S1. To improve gene expression in $S$. cerevisiae we optimized the codons CYP88D6 (OP-CYP88D6) and CYP72A154 (OPCYP72A154) for genes cloning into pUC57, which were synthesized by GenScript (GenScript, Nanjing, China). The optimized sequences of these are shown in the Additional file 1 .

We constructed pUC19L-Ptdh3-Tcyc1, pUC19L -Ppgk1-Tadh1, pUC19L-Ptef1-Tpgk1, and pUC19LPtef2-Tcyc1 utilizing seamless cloning and assembly by implementing the pEASY-UniSeamless Cloning and Assembly kit (TransGen Biotech, Beijing, China) (Additional file 1: Fig. S9a). The promoters of TDH3, PGK1, TEF1, and TEF2 as well as the terminators of CYC1, PGK1, and ADH1 were amplified from the genomic DNA of S. cerevisiae Cen.pk2-1D with the primers shown in Additional file 1: Table S1. Primers used for seamless cloning and assembly can be seen in Additional file 1: Table S2.

The workflow for construction and seamless cloning for the following: Ptef2-ERG19-cyc1, Ptdh3-CYP88D6Tcyc1, Ptdh3-OP-CYP88D6-Tcyc1, Ppgk1- $\beta$-AS-Tadh1, Ppgk1-tHMG1-Tadh1, Ptdh3-ERG20+9-Tcyc1, Ptdh3ERG9+20-Tcyc1, Ptef1-ERG1-Tpgk1, and Ptdh3AtCPR1-Tcyc1 (Additional file 1: Fig. S9b). The primers used for seamless cloning and assembly are shown in Additional file 1: Table S3.

To construct the expression cassettes for Pdth3-ERG8Ttdh3 and Ptdh3-ERG12-Ttdh3, ERG8 and ERG12 genes were amplified from the genomic DNA of $S$. cerevisiae Cen.pk2-1D then digested by SalI and NotI. After digestion they were then ligated into the SalI and NotI digested pATP424 by T4 DNA Ligase (Takara, Dalian, China). We used the cDNA of the G. uralensis to amplify GuCYB5 and ligate it into pATP424, the same as ERG8 forming the plasmid pATP424-GuCYB5. 
Table 1 Plasmids used in this study

\begin{tabular}{|c|c|c|}
\hline Name & Description & Source \\
\hline pUC19L & Cloning vector with multiple cloning sites, Amp & Invitrogen \\
\hline pATP423 & 2MICRON, HIS3 & Ishii et al. [38] \\
\hline pATP424 & 2MICRON, TRP1 & Ishii et al. [38] \\
\hline pATP425 & 2MICRON, LEU2 & Ishii et al. [38] \\
\hline pATP406 & W/o yeast origin, URA3 & Ishii et al. [38] \\
\hline PRS423 & 2MICRON, HIS3 & Christianson [39] \\
\hline pUC19L-P $P_{\text {tef1 }}-T_{p g k 1}$ & Cloning $P_{\text {tef } 1}-T_{p g k 1}$ cassette into pUC19L & This study \\
\hline $\mathrm{pUC19L-} P_{\text {tdh }}{ }^{-} \mathrm{T}_{\text {cyc1 }}$ & Cloning $P_{\text {tdh } 3}-T_{\text {cycl }}$ Cassette into pUC19L & This study \\
\hline pUC19L- $P_{p g k 1}-T_{a d h 1}$ & Cloning $P_{p g k 1}-T_{a d h 1}$ cassette into pUC19L & This study \\
\hline pUC19L- $P_{\text {tef2 }}-T_{\text {cyc1 }}$ & Cloning $P_{\text {tef2 }}-T_{\text {cyc1 }}$ cassette into pUC19L & This study \\
\hline pUC19L- $P_{t d h 3^{-}} T_{t d h 3}$ & Cloning $P_{\text {tdh }} 3^{-} T_{\text {tdh } 3}$ cassette into pUC19L & This study \\
\hline pUC19L-P tef2 - ERG19-T & Cloning $P_{\text {tef2 } 2}-E R G 19-T_{\text {cyc } 1}$ cassette into pUC19L & This study \\
\hline pUC19L- $P_{\text {tdh3 }}-E R G 9+20-T_{\text {cycl }}$ & Cloning $P_{\text {tdh } 3}-E R G 9+20-T_{\text {cyc }}$ cassette into pUC19L & This study \\
\hline pUC19L- $P_{\text {tdh3 }}-E R G 20+9-T_{\text {cycl }}$ & Cloning $P_{\text {tdh } 3}-E R G 20+9-T_{\text {cyc }}$ cassette into pUC19L & This study \\
\hline pUC19L- $P_{\text {tef1 }}-E R G 1-T_{p g k 1}$ & Cloning $P_{\text {tefl }}-E R G 1-T_{p g k 1}$ cassette into pUC19L & This study \\
\hline pUC19L-P $P_{p g k 1}-t H M G 1-T_{\text {adh } 1}$ & Cloning $P_{\text {pgk } 1}-$ tHMG1-T adh1 cassette into pUC19L & This study \\
\hline pUC19L-P $P_{p g k 1}-\beta-A S-T_{a d h 1}$ & Cloning $P_{p g k 1}-\beta-A S-T_{a d h 1}$ cassette into pUC19L & This study \\
\hline pUC19L- $P_{\text {tdh } 3}-C Y P 88 D 6-T_{\text {cycl }}$ & 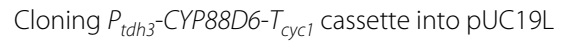 & This study \\
\hline pUC19L-P $P_{\text {tdh } 3}-A t C P R 1-T_{\text {cyc1 }}$ & Cloning $P_{\text {tdh } 3}-C P R 1-T_{\text {cycl }}$ cassette into pUC19L & This study \\
\hline pATP406-ERG8 & pATP406, expressing ERG8 gene by $\mathrm{P}_{\mathrm{TDH} 3}$ & This study \\
\hline PATP406-ERG10 & pATP406, expressing ERG10 gene by $\mathrm{P}_{\mathrm{ADH} 1}$ & This study \\
\hline PATP406-ERG12 & pATP406, expressing $E R G 12$ gene by $\mathrm{P}_{\mathrm{ADH} 1}$ & This study \\
\hline PATP406-ERG13 & pATP406, expressing $E R G 13$ gene by $\mathrm{P}_{\mathrm{ADH} 1}$ & This study \\
\hline pATP406-ID/1 & pATP406, expressing ID/1 gene by $P_{\text {PGK1 }}$ & This study \\
\hline pATP406-CYB5 & pATP406, expressing CY B5 gene by $\mathrm{P}_{\mathrm{TDH} 3}$ & This study \\
\hline PATP24- CYP72A154 & PATP424, expressing CYP72A154 gene by $P_{A D H 1}$ & This study \\
\hline pATP424-AtCPR1 & pATP424, expressing AtCPR1 gene by $\mathrm{P}_{\mathrm{TDH} 3}$ & This study \\
\hline pATP424- $\beta-A S$ & pATP424, expressing $\beta$-AS gene by $P_{\text {PGK1 }}$ & This study \\
\hline pATP424-OP-CYP72A154 & pATP424, expressing OP-CYP72A154 gene by $P_{A D H 1}$ & This study \\
\hline pATP424-OP88D6 & pATP424, expressing OP-CYP88D6 gene by $\mathrm{P}_{\mathrm{TDH} 3}$ & This study \\
\hline
\end{tabular}

The gene expression cassettes of Padh1-ERG10-Tadh1, Padh1-CYP72A154-Tadh1, Padh1-OP-CYP72A154Tadh1, and Ppgk1-IDI1-Tpgk1 were constructed similarly except that enzymatic digestion occurred with AvrII and FseI rather than SalI and NotI. Primers used for plasmid construction detection in Additional file 1: Table S4 and all plasmids used in study are shown in Table 1.

\section{Strain construction}

Saccharomyces cerevisiae Cen.pk2-1D, which was used as the parental strain, was purchased from EUROSCARF. To transform S. cerevisiae strains, we used the standard lithium acetate method or the electroporation method. Gene cassettes of Ppgk1- $\beta$-AS-Tadh, P tdh3-CYP88D6Tcyc1, Padh1-CYP72A154-Tadh1, and Ptdh3-AtCPR1Tcyc1 were amplified from the corresponding plasmids. Sequences of rDNA-up and rDNA-down were amplified from the DNA of S. cerevisiae Cen.pk2-1D using the primer set of GA-rDNA-up-F\&R and GA-rDNA-downF\&R. To amplify the sequence of Leu2 marker from pRS425 we used the primer set of GA-Leu2-F\&R. These six amplified DNA fragments were electroporated into $S$. cerevisiae Cen.pk2-1D using the DNA assembly method reported in previous studies [15] followed by selection on a SD-Leu plate. Similarly, fragments of Ppgk1- $\beta$ - $A S$-Tadh, Ptdh3-OP-CYP88D6-Tcyc1, Padh1-OP-CYP72A154Tadh1, Ptdh3-AtCPR1-Tcyc1, rDNA-up and rDNA-down were amplified and transferred into $S$. cerevisiae Cen. pk2-1D. We randomly selected colonies from the SD-Leu plates, after which six positive ones were verified. The highest GA-producing strain was designed as strain Y1.

Strain Y2 was constructed by integrating ERG20+9 (fused gene of ERG20 and ERG9 in ERG20+ERG9 way), $t H M G 1$, and ERG1 into the delta sites of Y1. Gene expression cassettes of Ptdh3-ERG20+9-Tcyc1, Ppgk1tHMG1-Tadh1, and Ptef1-ERG1-Tpgk1 were amplified 
from their corresponding plasmids. Delta-up and Deltadown were amplified from the DNA of $S$. cerevisiae Cen. pk2-1D using the primer set Delta-up-F\&R and Deltadown-F\&R. We amplified the Ura3 marker from pRS426 using the primer set of delta-URA-F\&R. After transferring these five fragments into strain $\mathrm{Y} 1$ we selected five positive colonies with the highest GA-producing strain designed as strain Y2. The construction of strain Y3 was similar to strain Y2 except $E R G 9+20$ (fused gene of ERG20 and ERG9 in ERG9+ERG20 way) was used in place of $E R G 20+9$.

By integrating ERG10,ERG8, and ERG13 into the YDR007W site of strain Y1, we created strain Y4. We amplified gene expression cassettes of ERG10, ERG8, and $E R G 13$ from plasmids of pATP406-ERG10, pATP406$E R G 8$, and pATP406-ERG12. The trp-up and trp-down sequences were amplified from DNA of $S$. cerevisiae Cen. pk2-1D using the primer set of Trp-up-F\&R and Trpdown-F\&R. The His marker was amplified from pRS423 using the primer set of Trp-his-F\&R. We electroporated these five fragments into stain $\mathrm{Y} 1$ and selected a positive colony from the SD-His plate, which we designed as strain $\mathrm{Y} 4$.

Strain Y5 was constructed by inserting ERG12, ERG8, ERG19, and IDI1 into the YPL069C site which was the BTS1 gene coding area. Gene expression cassettes of $E R G 12, E R G 8, E R G 19$, and IDI1 were amplified from plasmids of pATP406-ERG12, pATP406-ERG8, pUC19L$\mathrm{P}_{\text {tef2 }}-E R G 19-\mathrm{T}_{\text {cyc1 }}$, and pATP406-IDI1 using the primer set of BTS1-E12-F\&R, BTS1-E8-F\&R, BTS1-E19-F\&R, and BTS1-IDI1-F\&R. We amplified the BTS1-up and BTS1-down sequences from DNA of $S$. cerevisiae Cen. pk2-1D using the primer set of BTS1-up-F\&R and BTS1down-F\&R. We used the primer set of BTS1-Trp-F\&R to amplify the Trp marker from pATP424. These six fragments were transferred into strain $\mathrm{Y} 1$, after selection from SD-Trp plates.

Strain Y6 was constructed by integrating ERG20+9 (fused gene of ERG20 and ERG9 in ERG9+ERG20 way), tHMG1, and ERG1 into the delta sites of Y4. Fragments obtained were the same strain as in Y2 construction. Positive colonies were selected from SD-leu-his-ura plates and verified through PCR amplification and sequencing.

Strain $Y 7$ was constructed by integrating the gene cassettes of ERG12, GuCYB5, ERG19, and IDI1 into the YPL069C site of strain Y6. The gene expression cassette of $G u C Y B 5$ was amplified from pATP406-GuCYB5 using the primer set of BTS1-Cyb5-F and BTS1-Cyb5-R. Other fragments were obtained as mentioned in strain Y5 construction.

The genomic organization of these strains can be seen in Additional file 1: Fig. S10. Primers used for integration are shown in Additional file 1: Table S5 and all strains used are shown in Table 2.

\section{Yeast cultivation and medium}

To select positive colonies during engineering, we used an SD dropout medium supplemented with the corresponding dropout powder (SD-His-Leu-Trp-Ura). All strains were first pre-cultured into $15 \mathrm{~mL}$ culture tubes containing $2 \mathrm{~mL}$ medium, and grown at $30{ }^{\circ} \mathrm{C}, 250 \mathrm{rpm}$ and $\mathrm{OD}_{600}$ of approximately 1.0. Flasks $(50 \mathrm{~mL})$ containing $15 \mathrm{~mL}$ medium were then inoculated to an $\mathrm{OD}_{600}$ of 0.05 with these seed cultures. In the batch fermentation, strains (Cen.pK2-1D, Y1-Y7) were grown in YPD medium (yeast extract $20 \mathrm{~g} / \mathrm{L}$, tryptone $10 \mathrm{~g} / \mathrm{L}$ and glucose $20 \mathrm{~g} / \mathrm{L}$ ) at $30{ }^{\circ} \mathrm{C}$ under $250 \mathrm{rpm}$ for 6 days in the flask. After $144 \mathrm{~h}$ cultivation, cell density and metabolites concentration of all samples were measured. Flask

Table 2 Strains used in this study

\begin{tabular}{|c|c|c|}
\hline Name & Description & Source \\
\hline Cenpk2-1D & MATa ura3-52 trp1-289 leu2-3,112 his3 1 MAL2-8C SUC2 & EUROSCARF \\
\hline YO & $\begin{array}{l}P_{p g k 1}-\beta-A S-T_{a d h 1}, P_{t d h 3}-C Y P 88 D 6-T_{c y c 1}, P_{a d h 1}-C Y P 72 A 154-T_{a d h 1} \text { and } P_{t d h 3}-A t C P R 1-T_{c y c 1} \text { cassettes and LEU2 marker gene were } \\
\text { integrated into rDNA site of Cenpk2-1D }\end{array}$ & $\begin{array}{l}\text { This study } \\
\text { This study }\end{array}$ \\
\hline Y1 & $\begin{array}{l}P_{p g k 1}-\beta-A S-T_{\text {adh } 1}, P_{t d h 3}-O P C Y P 88 D 6-T_{c y c 1}, P_{a d h 1}-O P C Y P 72 A 154-T_{\text {adh } 1} \text { and } P_{t d h 3}-A t C P R 1-T_{c y c 1} \text { cassettes and LEU2 marker gene were } \\
\text { integrated into rDNA site of Cenpk2-1D }\end{array}$ & This study \\
\hline Y2 & $\begin{array}{l}P_{\text {tdh3 } 3}-E 20+9-T_{c y c 1}\left(P_{t d h 3}-E 20+9-T_{c y c 1}\right), P_{t e f 1}-E R G 1-T_{p g k 1} \text { and } P_{p g k 1}-t H M G 1-T_{a d h 1} \text { cassettes and URA } A_{3} \text { marker gene were integrated } \\
\quad \text { into delta site of } Y 1\end{array}$ & This study \\
\hline Y4 & $P_{t d h 3}-E R G 8-T_{t d h 3}, P_{a d h 1}-E 13-T_{a d h 1}$ and $P_{a d h 1}-E 10-T_{a d h 1}$ cassettes and HIS3 marker gene were integrated into TRP1 site of Y1 & This study \\
\hline Y5 & $\begin{array}{l}P_{a d h 1}-E R G 12-T_{a d h 1}, P_{t d h 3}-C y b 5-T_{t d h 3}, P_{t e f 2}-E R G 19-T_{c y c 1} \text { and } P_{p g k 1}-I D I-T_{p g k 1} \text { cassettes and HIS3 marker gene were integrated into } \\
\text { BTS1 site of } Y 1\end{array}$ & This study \\
\hline Y6 & $\begin{array}{l}P_{\text {tdh3 } 3}-E 20+9-T_{c y c 1}\left(P_{t d h 3}-E 20+9-T_{c y c 1}\right), P_{t e f 1}-E R G 1-T_{p g k 1} \text { and } P_{p g k 1}-t H M G 1-T_{a d h 1} \text { cassettes and URA3 marker gene were integrated } \\
\quad \text { into delta site of } Y 4\end{array}$ & This study \\
\hline Y7 & $\begin{array}{l}P_{a d h 1}-E R G 12-T_{a d h 1}, P_{t d h 3^{-}}-C y b 5-T_{t d h{ }_{3}}, P_{t e f 2}-E R G 19-T_{c y c 1} \text { and } P_{p g k 1}-1 D I-T_{p g k 1} \text { cassettes and HIS3 marker gene were integrated into } \\
\text { BTS1 site of } Y 6\end{array}$ & This study \\
\hline
\end{tabular}


fermentation results are presented as the mean \pm S.D. of at least three independent experiments.

The fermentation medium was composed of glucose $(19.5 \mathrm{~g} / \mathrm{L}),(\mathrm{NH} 4)_{2} \mathrm{SO}_{4}(15 \mathrm{~g} / \mathrm{L}), \mathrm{KH}_{2} \mathrm{PO} 4(8 \mathrm{~g} / \mathrm{L})$, $\mathrm{MgSO}_{4} \cdot 7 \mathrm{H}_{2} \mathrm{O}(6.15 \mathrm{~g} / \mathrm{L})$, vitamin solution $(12 \mathrm{~mL} / \mathrm{L})$, and a trace metal solution $(10 \mathrm{~mL} / \mathrm{L})$. The trace metal solution contained: $\mathrm{ZnSO}_{4} \cdot 7 \mathrm{H}_{2} \mathrm{O}(5.75 \mathrm{~g} / \mathrm{L}), \mathrm{MnCl}_{2} \cdot 4 \mathrm{H}_{2} \mathrm{O}$ $(0.32 \mathrm{~g} / \mathrm{L})$, anhydrous $\mathrm{CuSO}_{4}(0.32 \mathrm{~g} / \mathrm{L}), \mathrm{CoCl}_{2} \cdot 6 \mathrm{H}_{2} \mathrm{O}$ $(0.47 \mathrm{~g} / \mathrm{L}), \quad \mathrm{Na}_{2} \mathrm{MoO}_{4} \cdot 2 \mathrm{H}_{2} \mathrm{O}(0.48 \mathrm{~g} / \mathrm{L}), \quad \mathrm{CaCl}_{2} \cdot 2 \mathrm{H}_{2} \mathrm{O}$ $(2.9 \mathrm{~g} / \mathrm{L}), \mathrm{FeSO}_{4} \cdot 7 \mathrm{H}_{2} \mathrm{O}(2.8 \mathrm{~g} / \mathrm{L}), 0.5 \mathrm{M}$ EDTA $(80 \mathrm{~mL} / \mathrm{L})$. The vitamin solution contained: biotin $(0.05 \mathrm{~g} / \mathrm{L})$, calcium pantothenate $(1 \mathrm{~g} / \mathrm{L})$, nicotinic acid $(1 \mathrm{~g} / \mathrm{L})$, myo-inositol $(25 \mathrm{~g} / \mathrm{L})$, thiamine $\mathrm{HCl}(1 \mathrm{~g} / \mathrm{L})$, pyridoxal $\mathrm{HCl}(1 \mathrm{~g} / \mathrm{L})$, and $p$-aminobenzoic acid $(0.2 \mathrm{~g} / \mathrm{L})$. The medium used in fermentation was based on media described previously $[15$, 17].

Strain Y7 was used for the production of GA through fed-batch fermentation. Seed culture was prepared by inoculating several colonies into a $250 \mathrm{~mL}$ flask containing $50 \mathrm{~mL}$ culture medium, and incubating at $30^{\circ} \mathrm{C}$ at $250 \mathrm{rpm}$ for $24 \mathrm{~h}$. Fed-batch fermentation was conducted in a $7.5 \mathrm{~L}$ bioreactor with $3 \mathrm{~L}$ fermentation media. The size of the inoculum was $5 \%$. The fermentation conditions were set at $30^{\circ} \mathrm{C}$, with a $\mathrm{pH}=6$, controlled by $10 \mathrm{M}$ $\mathrm{NaOH}$. We intermittently added $800 \mathrm{~g} / \mathrm{L}$ of glucose to maintain a $30 \mathrm{~g} / \mathrm{L}$ concentration at 12, 24, 36, 48 and $72 \mathrm{~h}$ time points.

\section{Analysis}

Optical densities at $600 \mathrm{~nm}\left(\mathrm{OD}_{600}\right)$ of all strains after 6 days fermentation were measured using a Shimadzu UV-2550 spectrophotometer.

Yeast cells after 6 days fermentation were collected by centrifugation at $10,000 \mathrm{~g}$ for $5 \mathrm{~min}$ and crushed using Bead Beater (BioSpec, USA) after which ultrasonic extraction with $2 \mathrm{~mL}$ extraction solutions (acetone:methanol=1:1) occurred 2 times. The extracts were trimethylsilylated with $\mathrm{N}$-methyl- $\mathrm{N}$-trimethylsilyltrifluoroacetamide (Sigma-Aldrich) at $80^{\circ} \mathrm{C}$ for $30 \mathrm{~min}$.

The samples were analyzed by an Agilent Technology $7890 \mathrm{GC}$, coupled with a $7000 \mathrm{C}$ Triple Quadrupole MS (Agilent Technologies, Santa Clara, CA USA). Two microliters of the sample was injected into splitless mode with an injector temperature of $300{ }^{\circ} \mathrm{C}$. Two HP-5 ms columns (Agilent Technologies, Santa Clara, CA USA), $15 \mathrm{~m} * 0.25 \mathrm{~mm}$ i.d. ${ }^{*} 0.25 \mu \mathrm{m}$ film thickness, were connected by a purged ultimate union (PUU) to provide sample separation. A pneumatics control module to implement backflush during post-run procedures controlled the PUU. Helium carrier gas was held at a constant flow for two columns with the first column set at a flow rate of $1.1 \mathrm{~mL} / \mathrm{min}$ and the second at a flow rate of $1.3 \mathrm{~mL} / \mathrm{min}$. The column temperature program was as follows: 1 min of $80^{\circ} \mathrm{C}$ followed by heating the column to $310{ }^{\circ} \mathrm{C}$ at $20^{\circ} \mathrm{C} / \mathrm{min}$ and maintaining $310^{\circ} \mathrm{C}$ for $17.5 \mathrm{~min}$. During post-run, the oven temperature was set at $310{ }^{\circ} \mathrm{C}$ for $7 \mathrm{~min}$, while the injector pressure was decreased to 2 psi and the pressure of the PUU increased to $55 \mathrm{psi}$ to backflush the less volatile matrix components of the first column.

The MS transfer line and ion source temperatures were set to $300{ }^{\circ} \mathrm{C}$ and $280{ }^{\circ} \mathrm{C}$, respectively. The Quadrupole temperature was $\mathrm{Q} 1=\mathrm{Q} 2=150^{\circ} \mathrm{C}$ and solvent delay was $5 \mathrm{~min}$.

For quantification, we collected data in multiple reaction monitoring (MRM) mode using squalene, lanosterol, ergosterol, $\beta$-amyrin and glycyrrhetinic acid standards (all purchased from Sigma Aldrich). Two MRM transitions (one for quantification and the other for qualification) were optimized for each metabolite and are shown in Additional file 1: Table S7.

\section{Additional file}

Additional file 1. Additional figures and tables

\section{Acknowledgements}

The authors gratefully thank Dr. Wenjun Huang at Wuhan Botanical Garden, Chinese Academy of Sciences for the gift of plasmids pATP424, 406. We also thank professor Yujun Zhang for his useful suggestions and support.

\section{Authors' contributions}

CW performed all the experiments, statistical analysis, and drafted the manuscript. XS, MS and MZ performed the vector and strain construction. JW participated in the chemical analysis. YW, JX and XL participated in its design and coordination.WS and SC directly supervised the experimental design, execution, data analysis and manuscript writing. All authors read and approved the final manuscript.

\section{Funding}

This research was supported by the National Natural Science Foundation of China (No. 81503196) and by the National Key R\&D Program of China (2018YFC1706500) and by the Institute of Chinese Materia Medic (L2015018).

\section{Availability of data and materials}

All data generated or analyzed during this study are included in this published article and its Additional file 1.

\section{Ethics approval and consent to participate} Not applicable.

\section{Consent for publication}

The authors are consent for publication.

\section{Competing interests}

The authors declare that they have no competing interests.

\section{Author details}

${ }^{1}$ Key Laboratory of Beijing for Identification and Safety Evaluation of Chinese Medicine, Institute of Chinese Materia Medica, China Academy of Chinese Medical Sciences, No. 16 Nanxiaojie, Dongzhimennei Ave, Beijing 100700, People's Republic of China. ${ }^{2}$ School of Life Science, Huai Bei Normal University, Huaibei 23500, People's Republic of China. ${ }^{3}$ School of Chemistry, Chemical Engineering and Life Sciences, Wuhan University of Technology, Wuhan 430070, People's Republic of China. ${ }^{4}$ Agilent Technologies (China) Co., 
Ltd., Wangjingbei Road, Chaoyang District, Beijing 100102, China. ${ }^{5}$ National Key Laboratory of Biochemical Engineering, Institute of Process Engineering, Chinese Academy of Sciences, Beijing 100190, People's Republic of China. ${ }^{6}$ Key Laboratory of South China Agricultural Plant Molecular Analysis and Genetic Improvement, Provincial Key Laboratory of Applied Botany, South China Botanical Garden, Chinese Academy of Sciences, Guangzhou 510650, People's Republic of China.

\section{Received: 31 January 2019 Accepted: 14 May 2019} Published online: 28 May 2019

\section{References}

1. Nomura T, Fukai T, Akiyama T. Chemistry of phenolic compounds of licorice (Glycyrrhiza species) and their estrogenic and cytotoxic activities. Pure Appl Chem. 2002;74:1199-206.

2. Cinatl J, Morgenstern B, Bauer G, Chandra P, Rabenau H, Doerr HW. Glycyrrhizin, an active component of liquorice roots, and replication of SARS-associated coronavirus. Lancet. 2003;361:2045-6.

3. Asl MN, Hosseinzadeh H. Review of pharmacological effects of Glycyrrhiza sp. and its bioactive compounds. Phytother Res. 2008;22:709-24.

4. Finney RS, Somers GF. The antiinflammatory activity of glycyrrhetinic acid and derivatives. J Pharm Pharmacol. 2011;10:613-20.

5. Fiore C, Eisenhut M, Krausse R, Ragazzi E, Pellati D, Armanini D, Bielenberg J. Antiviral effects of Glycyrrhiza species. Phytother Res. 2008;22:141-8.

6. Kroes BH, Beukelman CJ, Aj VDB, Wolbink GJ, Van DH, Labadie RP. Inhibition of human complement by beta-glycyrrhetinic acid. Immunology. 1997;90:115-20.

7. Abe N, Ebina T, Ishida N. Interferon induction by glycyrrhizin and glycyrrhetinic acid in mice. Microbiol Immunol. 1982;26:535-9.

8. Hayashi $\mathrm{H}$, Sudo $\mathrm{H}$. Economic importance of licorice. Plant Biotechnol. 2009;26:101-4.

9. Seki H, Ohyama K, Sawai S, Mizutani M, Ohnishi T, Sudo H, Akashi T, Aoki T, Saito K, Muranaka T. Licorice $\beta$-amyrin 11-oxidase, a cytochrome P450 with a key role in the biosynthesis of the triterpene sweetener glycyrrhizin. Proc Natl Acad Sci USA. 2008;105:14204-9.

10. Guo J, Zhou YJ, Hillwig ML, Shen Y, Yang L, Wang Y, Zhang X, Liu W, Peters RJ, Chen X. CYP76AH1 catalyzes turnover of miltiradiene in tanshinones biosynthesis and enables heterologous production of ferruginol in yeasts. Proc Natl Acad Sci USA. 2013;110:12108-13.

11. Han JY, Hwang HS, Choi SW, Kim HJ, Choi YE. Cytochrome P450 CYP716A53v2 catalyzes the formation of protopanaxatriol from protopanaxadiol during ginsenoside biosynthesis in Panax ginseng. Plant Cell Physiol. 2012;53:1535-45.

12. Moses T, Pollier J, Almagro L, Buyst D, Van MM, Pedreño MA, Martins JC, Thevelein JM, Goossens A. Combinatorial biosynthesis of sapogenins and saponins in Saccharomyces cerevisiae using a C-16a hydroxylase from Bupleurum falcatum. Proc Natl Acad Sci USA. 2014;111:1634-9.

13. Paddon CJ, Westfall PJ, Pitera DJ, Benjamin K, Fisher K, McPhee D, Leavell MD, Tai A, Main A, Eng D, Polichuk DR, Teoh KH, Reed DW, Treynor T, Lenihan J, Fleck M, Bajad S, Dang G, Dengrove D, Diola D, Dorin G, Ellens KW, Fickes S, Galazzo J, Gaucher SP, Geistlinger T, Henry R, Hepp M, Horning T, lqba T, Jiang H, Kizer L, Lieu B, Melis D, Moss N, Regentin R, Secrest S, Tsuruta H, Vazquez R, Westblade LF, Xu L, Yu M, Zhang Y, Zhao L, Lievense J, Covello PS, Keasling JD, Reiling KK, Renninger NS, Newman JD. High-level semi-synthetic production of the potent antimalarial artemisinin. Nature. 2013;496:528-32.

14. Westfall PJ, Pitera DJ, Lenihan JR, Eng D, Woolard FX, Regentin R, Horning T, Tsuruta $\mathrm{H}$, Melis DJ, Owens A. Production of amorphadiene in yeast, and its conversion to dihydroartemisinic acid, precursor to the antimalarial agent artemisinin. Proc Natl Acad Sci USA. 2012;109:655-6.

15. Dai Z, Liu Y, Zhang X, Shi M, Wang B, Wang D, Huang L, Zhang X. Metabolic engineering of Saccharomyces cerevisiae for production of ginsenosides. Metab Eng. 2013;20:146-56.

16. Yan X, Fan Y, Wei W, Wang P, Liu Q, Wei Y, Zhang L, Zhao G, Yue J, Zhou Z. Production of bioactive ginsenoside compound $\mathrm{K}$ in metabolically engineered yeast. Cell Res. 2014;24:770-3.
17. Wang P, Wei Y, Fan Y, Liu Q, Wei W, Yang C, Zhang L, Zhao G, Yue J, Yan $X$. Production of bioactive ginsenosides Rh2 and Rg3 by metabolically engineered yeasts. Metab Eng. 2015;29:97-105.

18. Leonard E, Ajikumar PK, Thayer K, Xiao WH, Mo JD, Tidor B, Stephanopoulos G, Prather KLJ. Combining metabolic and protein engineering of a terpenoid biosynthetic pathway for overproduction and selectivity control. Proc Natl Acad Sci USA. 2010;107:13654-9.

19. Qu Y, Easson ML, Froese J, Simionescu R, Hudlicky T, De LV. Completion of the seven-step pathway from tabersonine to the anticancer drug precursor vindoline and its assembly in yeast. Proc Natl Acad Sci USA. 2015;112:6224-9.

20. Zhou YJ, Wei G, Rong Q, Jin G, Chu H, Liu W, Wei Y, Zhu Z, Li G, Zhu G. Modular pathway engineering of diterpenoid synthases and the mevalonic acid pathway for miltiradiene production. J Am Chem Soc. 2012;134:3234-41.

21. Guo J, Ma X, Cai Y, Ma Y, Zhan Z, Zhou YJ, Liu W, Guan M, Yang J, Cui G. Cytochrome P450 promiscuity leads to a bifurcating biosynthetic pathway for tanshinones. New Phytol. 2016;210:525-34.

22. Brown S, Clastre M, Courdavault V, O'Connor SE. De novo production of the plant-derived alkaloid strictosidine in yeast. Proc Natl Acad Sci USA. 2015;112:3205-10.

23. Bai Y, Yin H, Bi H, Zhuang Y, Liu T, Ma Y. De novo biosynthesis of gastrodin in Escherichia coli. Metab Eng. 2016;35:138-47.

24. Hayashi H, Huang P, Kirakosyan A, Inoue K, Hiraoka N, Ikeshiro Y, Kushiro T, Shibuya M, Ebizuka Y. Cloning and characterization of a CDNA encoding beta-amyrin synthase involved in glycyrrhizin and soyasaponin biosyntheses in licorice. Biol Pharm Bull. 2001;24:912-6.

25. Seki H, Muranaka T. Triterpene functional genomics in licorice for identification of CYP72A154 involved in the biosynthesis of glycyrrhizin. Plant Cell. 2011;23:4112-3.

26. Xu G, Cai W, Gao W, Liu C. A novel glucuronosyltransferase has an unprecedented ability to catalyse continuous two-step glucuronosylation of glycyrrhetinic acid to yield glycyrrhizin. New Phytol. 2016;212:123-35.

27. Kirby J, Romanini DW, Paradise EM, Keasling JD. Engineering triterpene production in Saccharomyces cerevisiae beta-amyrin synthase from Artemisia annua. FEBS J. 2010;275:1852-9.

28. Zhang G, Cao Q, Liu J, Liu B, Li J, Li C. Refactoring $\beta$-amyrin synthesis in Saccharomyces cerevisiae. AIChE J. 2015;61:3172-9.

29. Zhu M, Wang $C$, Sun W, Zhou A, Wang Y, Zhang G, Zhou X, Huo Y, Li C. Boosting 11-oxo- $\beta$-amyrin and glycyrrhetinic acid synthesis in Saccharomyces cerevisiae via pairing novel oxidation and reduction system from legume plants. Metab Eng. 2017;45:43-50.

30. Galanie S, Thodey K, Trenchard IJ, Interrante MF, Smolke CD. Complete biosynthesis of opioids in yeast. Science. 2015;349:1095-100.

31. Thodey K, Galanie S, Smolke CD. A microbial biomanufacturing platform for natural and semisynthetic opioids. Nat Chem Biol. 2014;10:837-44.

32. Ajikumar PK, Xiao WH, Tyo KEJ, Wang Y, Simeon F, Leonard E, Mucha O, Phon TH, Pfeifer B, Stephanopoulos G. Isoprenoid pathway optimization for taxol precursor overproduction in Escherichia coli. Science. 2010;330:70-4.

33. Li Y, Pfeifer BA. Heterologous production of plant-derived isoprenoid products in microbes and the application of metabolic engineering and synthetic biology. Curr Opin Plant Biol. 2014;19:8-13.

34. Zhao F, Bai P, Liu T, Li D, Zhang X, Lu W, Yuan Y. Optimization of a cytochrome $\mathrm{P} 450$ oxidation system for enhancing protopanaxadiol production in Saccharomyces cerevisiae. Biotechnol Bioeng. 2016;113:1787-95.

35. Renault H, Bassard JE, Hamberger B, Werckreichhart D. Cytochrome P450-mediated metabolic engineering: current progress and future challenges. Curr Opin Plant Biol. 2014;19:27-34.

36. Zhang H, Im SC, Waskell L. Cytochrome b5 increases the rate of product formation by cytochrome P450 2B4 and competes with cytochrome $\mathrm{P} 450$ reductase for a binding site on cytochrome P450 2B4. J Biol Chem. 2007;282:29766-76.

37. Zhou K, Qiao K, Edgar S, Stephanopoulos G. Distributing a metabolic pathway among a microbial consortium enhances production of natural products. Nat Biotechnol. 2015;33:377-83. 
38. Ishii J, Kondo T, Makino H, Ogura A, Matsuda F, Kondo A. Three gene expression vector sets for concurrently expressing multiple genes in Saccharomyces cerevisiae. FEMS Yeast Res. 2014;14:399.

39. Christianson TW, Sikorski RS, Dante M, Shero JH, Hieter P. Multifunctional yeast high copy number shuttle vectors. Gene. 1992;110:119-22.

\section{Publisher's Note}

Springer Nature remains neutral with regard to jurisdictional claims in published maps and institutional affiliations.
Ready to submit your research? Choose BMC and benefit from:

- fast, convenient online submission

- thorough peer review by experienced researchers in your field

- rapid publication on acceptance

- support for research data, including large and complex data types

- gold Open Access which fosters wider collaboration and increased citations

- maximum visibility for your research: over $100 \mathrm{M}$ website views per year

At BMC, research is always in progress.

Learn more biomedcentral.com/submissions 